\title{
Was soll und kann die Europäische Union?
}

\author{
von Fritz W. Scharpf
}

Die nachfolgend wiedergegebene Präsentation informiert über einen Vortrag, den der Autor auf Einladung des Nomos-Verlags und des Internationalen Instituts für Staats- und Europawissenschaften (ISE) anlässlich eines Kolloquiums zum Thema „,Nationalstaat und Europäische Union: eine Bestandsaufnahme" am 30. November 2012 auf Schloss Leopoldskron in Salzburg hielt. Den zahlreichen Nachfragen kommen der Autor und die Schriftleitung mit dieser komprimierten Darstellung nach; ein die gesamte Veranstaltung zusammenfassender Bericht erscheint ebenfalls in diesem Heft.

The following presentation comprises a lecture given by the author at a colloquium on „The Nation-State and the European Union: Taking Stock“, held at Schloss Leopoldskron (Salzburg) on 30 November 2012 at the invitation of Nomos Publishers and the International Institute for Comparative Government and European Policy (ISE). By presenting these slides, the author and the journal respond to various requests for publication; $a$ brief report commenting upon the entirety of the proceedings is part of this issue.

\section{Was soll die EU? \\ - Erster Diskurs -}

- Integration als Wert an sich:

Verwirklichung einer immer engeren Union der Völker Europas (Art. 1 EUV)

- Friedensordnung

- Überwindung des Nationalismus: Normative Abwertung des Nationalstaats

$\rightarrow$ Je mehr Europa, desto besser 


\section{Was soll die EU? \\ - Zweiter Diskurs -}

- Integration im Interesse der Mitgliedstaaten:

Subsidiarität (Art. 5 Abs. 3 EUV)

- Aufgaben, die Mitgliedstaaten nicht bewältigen

- Globalisierung $\rightarrow$ Zunehmende Interdependenz

- Sinkende Effektivität/Legitimität nationaler Politik

- Politische Handlungsfähigkeit durch Integration

- Aber: Integration erzeugt auch Interdependenz und beschränkt Handlungsfähigkeit der Staaten

\section{Was kann die EU? \\ - Erste Schranke: Hoher Konsensbedarf -}

- Heterogenität der Mitglieder:

ökonomisch, kulturell, institutionell, politisch

- Schwache kollektive Identität $\rightarrow$ begrenzte Legitimität majoritärer Konfliktregelung

- EU-Politik erfordert deshalb

- entweder breiten Konsens: community method

- oder Entpolitisierung: EuGH/Komitologie

$\rightarrow$ Politische Handlungsfähigkeit ist beschränkt 


\section{Was kann die EU?}

- Zweite Schranke: Geringe Lernfähigkeit -

- Hoher Konsensbedarf der EU-Politik:

behindert Revision des einmal Beschlossenen

- Suprematie des EU-Rechts:

verhindert Innovation auf der nationalen Ebene

- Integration als Selbstzweck:

tabuisiert Rückschritte

$\rightarrow$ Reaktion auf Fehler: „mehr Europa“

\section{Extremfall: Währungsunion erzeugt Probleme, die sie nicht lösen kann}

- Monetäre Integration heterogener Ökonomien

- Wegfall des Wechselkurses \& der Zahlungsbilanz

- Einheitliche monetäre Politik

- Beseitigt nationale Steuerungskompetenz

- Zentrale Steuerung: „one size fits none“

- Für Deutschland zu restriktiv: Rezession

- Für GIPSI-Länder zu expansiv: kreditfinanzierte Überkonjunktur

- 1999-2008: Dynamische Fehlentwicklung 


\section{Angleichung der Nominalzinsen}

10-year government bond yields (nominal)

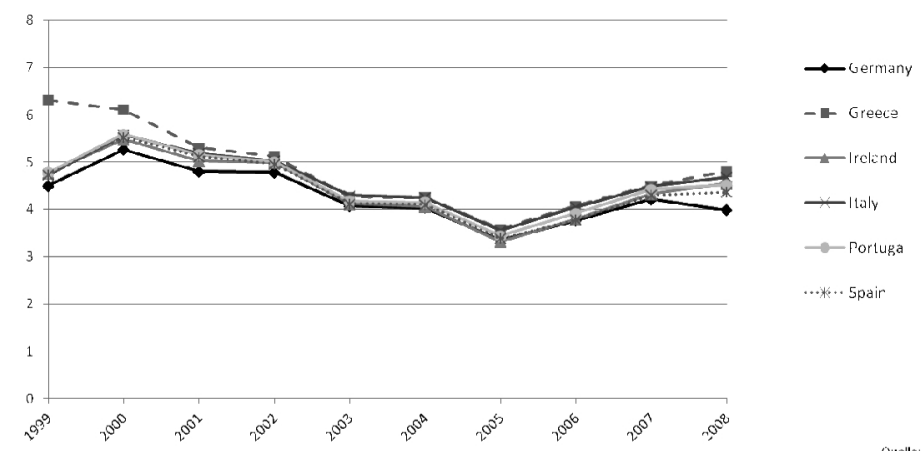

Quelle: OECD

\section{Unterschiedliche Wachstumsraten}

GDP growth rate (volume)

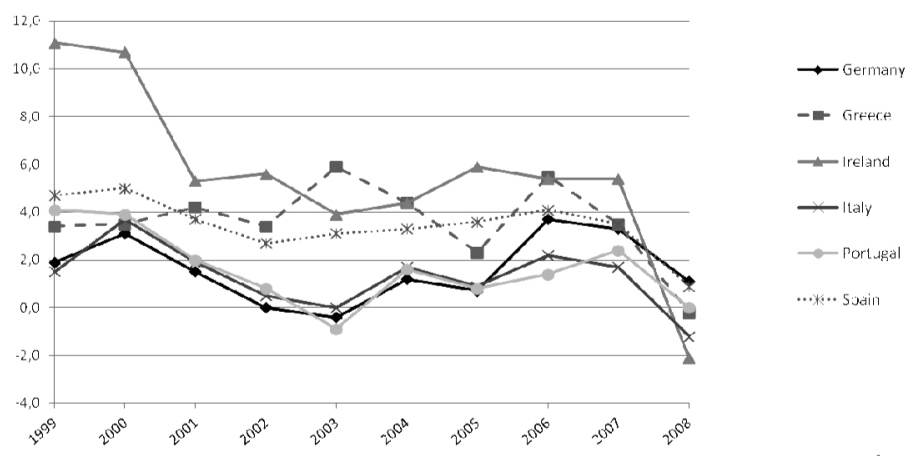




\section{Divergierende Lohnstückkosten}

unit labour costs (nominal, 1999=100)

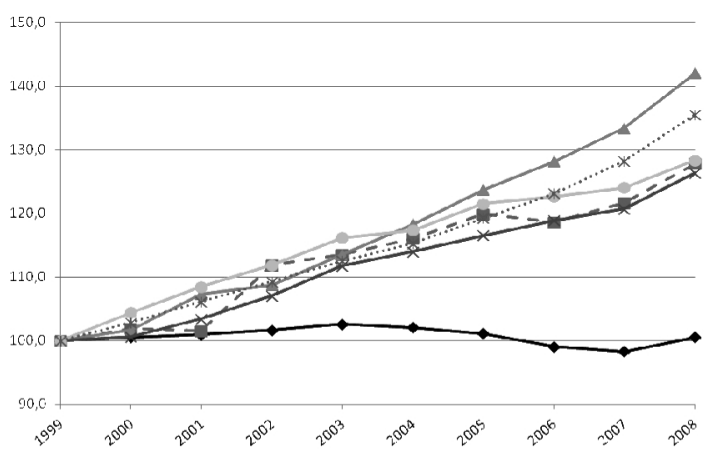

\section{Divergierende reale Wechselkurse}

real effective exchange rates (ULC, rel. to EA16, 1999=100)

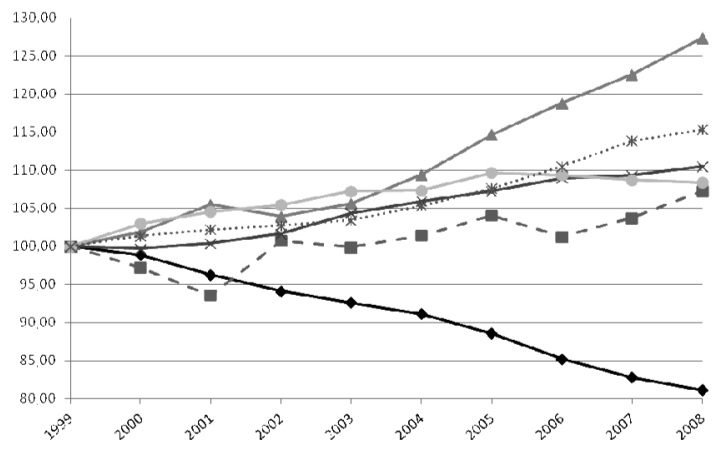




\section{Divergierende Leistungsbilanzen}

current account (in \% GDP)
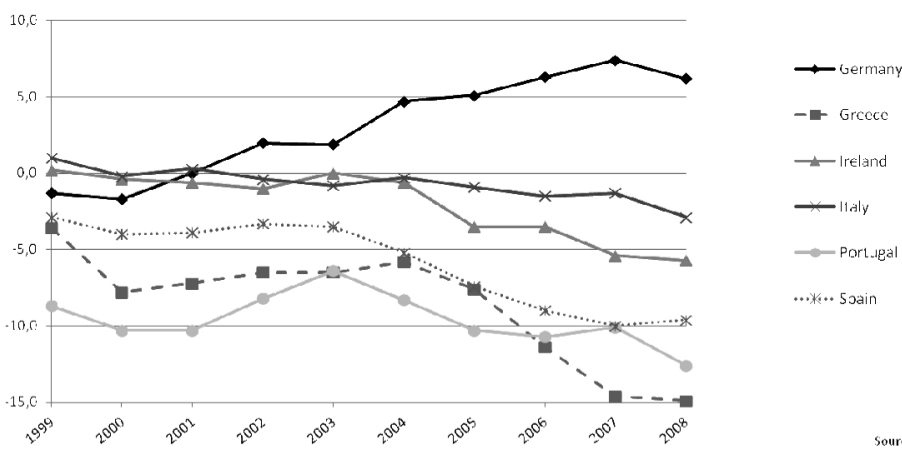

- Grecce

$\rightarrow$ Ireland

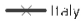

$\rightarrow$ Porluga

\section{Kompensation durch Kapitalimporte}

financial balance in \% GDP
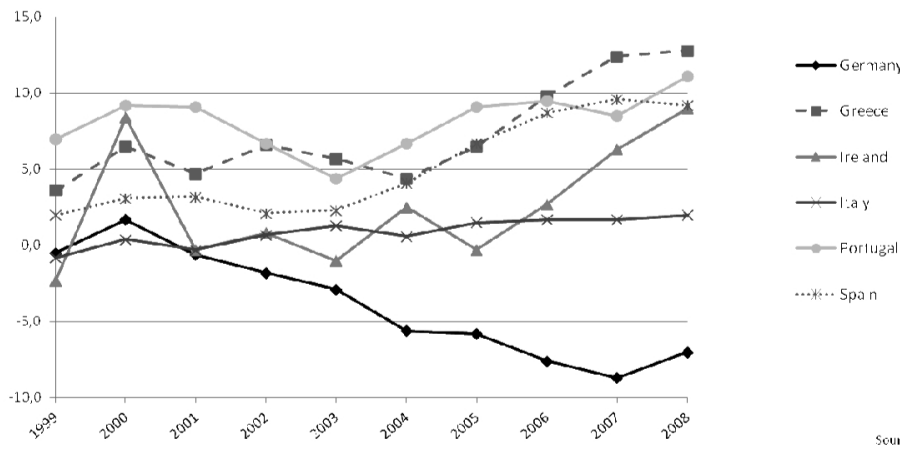

- - Greect

$\rightarrow$ Ireand

$\longrightarrow$ - lay

$\longrightarrow$ Forlugit 


\section{Keine Dynamik der Staatsschulden}

government consolidated gross debt in \% GDP

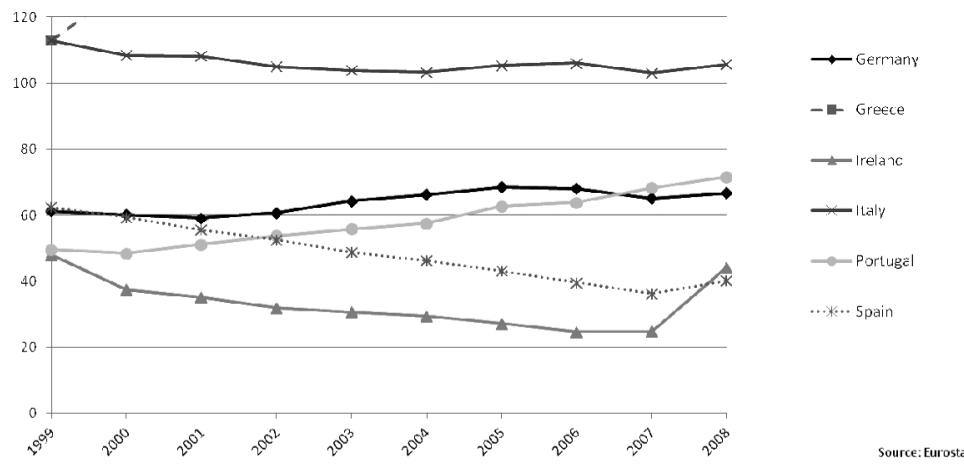

\section{8: Lehman-Finanzkrise $\rightarrow$ Eurokrise}

- Weltweite Kreditverknappung; besonders betroffen: kreditabhängige Länder

- Bankenrettung $\rightarrow$ Staatsverschuldung überall

- Risikozinsen steigen in Euroländern mit Leistungsbilanzdefizit

- Drohende Staatsinsolvenz $\rightarrow$ Eurokrise

- Aber auch: Wirtschafts- und Sozialkrise

- Verlorene Wettbewerbsfähigkeit 


\section{Ab 2008: steigende Staatsverschuldung}

government consolidated gross debt in \% GDP

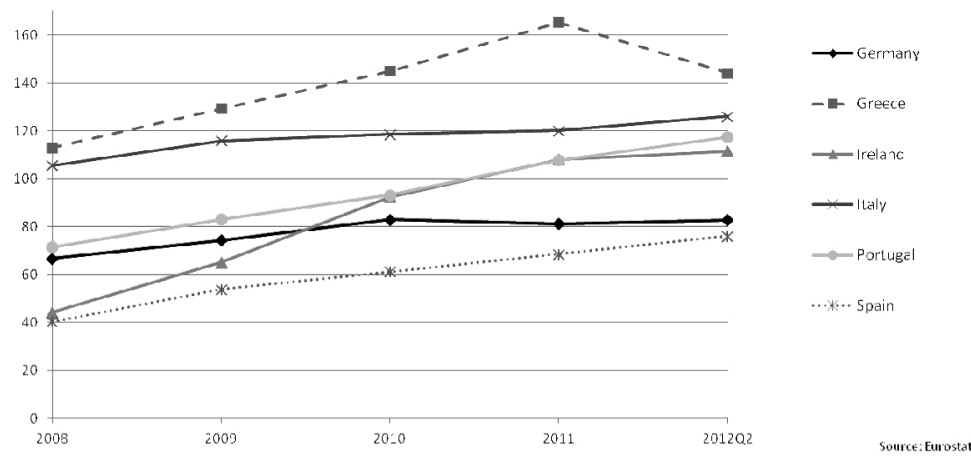

\section{Ab 2008: Risikozinsen der GIPSI- Staaten}

10-year government bond yields (nominal)

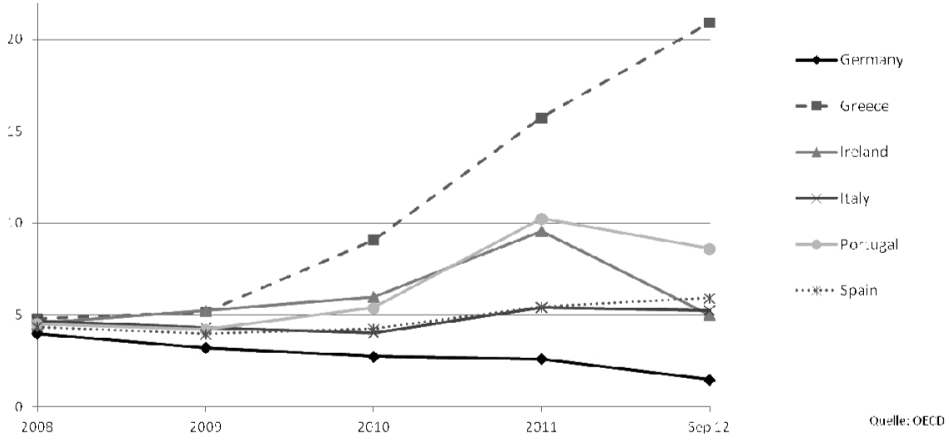




\section{Wirtschafts- und Sozialkrise der GIPSI- Staaten}

unemployment rates

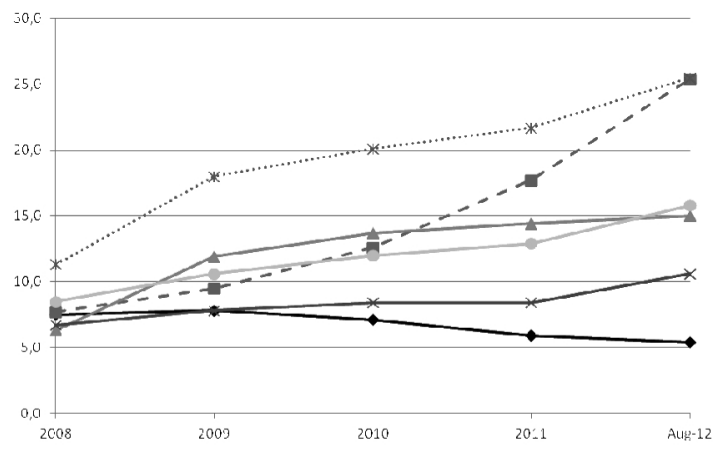

\section{Erste Reaktion auf die Eurokrise}

- Euro-Rettung um jeden Preis:

- Euro-Rettungsfonds trotz bailout-Verbots

- Später: Indirekte Staatsfinanzierung durch EZB

- Aber falsche Diagnose:

Staatsverschuldung als Ursache der Krise

- Deshalb zunächst falsche Therapie:

- rigorose Sparauflagen verschärfen die Krise

- EZB-Intervention hilft gegen Staatsinsolvenz

- aber: Wirtschafts- und Sozialkrise dauert an 


\section{1-12: Reformen der Währungsunion}

- Fiskalpakt und sixpack-Defizitverfahren:

- verschärfte Defizit- und Schuldenregeln für nationale Haushalte

- frühzeitige Aufsicht durch Kommission

- quasi-automatische Sanktionen

- Sixpack-Kontrolle makroökonomischer Ungleichgewichte:

- scoreboard und Indikatoren

- Empfehlungen der Kommission ohne Regeln und ohne Kompetenzgrenzen

- quasi-automatische Sanktionen

\section{Logik der bisherigen Reformen}

- Die monetäre Zentralisierung bleibt bestehen aber: ökonomische Heterogenität wird jetzt anerkannt

- Trotzdem:

- keine differenzierte europäische Geldpolitik

- keine antizyklische europäische Fiskalpolitik

- kein Spielraum für nationale Fiskalpolitik

- Stattdessen:

- Versuch der Zentralisierung der nationalen Mikropolitik

- Aber:

- dafür gibt es keine Theorie

- dafür kann es keine Regeln geben

- dafür fehlt der Kommission die politische Legitimation 


\section{Währungsunion: Was soll und kann die EU?}

- Monetäre Integration heterogener Ökonomien

- Zentralisierte Makropolitik erzeugt Krisen

- Die Maxime „mehr Europa“ tabuisiert die Revision

- Reaktion: Weitere Zentralisierung der Mikropolitik

- Ziel: Homogenisierung der Euro-Ökonomien

- Nicht erreichbar durch autonome Marktmechanismen

- Nicht erreichbar durch nationale demokratische Politik

- Durchsetzung erfordert europäischen Zwang

- Ökonomische Effektivität zweifelhaft

- Politische Legitimation ungesichert

- Fazit: Die EWU versucht mehr als sie kann und soll 